\title{
Electromagnetic Form Factors of the $\Lambda(1405)$ in (2+1)-flavour Lattice QCD
}

\author{
Benjamin J. Menadue*, Waseem Kamleh, Derek B. Leinweber, M. Selim Mahbub, \\ Benjamin J. Owen \\ Special Research Centre for the Subatomic Structure of Matter \\ School of Chemistry \& Physics, University of Adelaide, SA, 5005, Australia \\ E-mail: ben.menadue@adelaide.edu.au \\ waseem.kamleh@adelaide.edu.au \\ derek.leinweber@adelaide.edu.au \\ md.mahbub@adelaide.edu.au \\ benjamin.owen@adelaide.edu.au
}

The $\Lambda(1405)$ is unusual in that it lies surprisingly low in mass. At $1405.1 \mathrm{MeV}$, it lies lower than the lowest-lying odd-parity state of the nucleon, even though it contains a valence strange quark. We build on our recent success in isolating this otherwise elusive state in lattice QCD using correlation matrix techniques coupled with source and sink smearing, and present first results from our world-first calculation of the electromagnetic form factors of this unusual state. We use the PACS-CS (2+1)-flavour full-QCD ensembles, available through the ILDG.

The 30th International Symposium on Lattice Field Theory

June 24-29, 2012

Cairns, Australia

\footnotetext{
* Speaker.
} 


\section{Introduction}

The lowest-lying odd-parity state of the $\Lambda$ baryon, has an unusually low mass of $1405.1_{-1.0}^{+1.3} \mathrm{MeV}$ [1]. Indeed, the $\Lambda(1405)$ lies lower than the lowest-lying odd-parity nucleon state, even though it possesses a valence strange quark. Consequently, there has been much interest over the years in the internal structure of this unusual state.

While many previous lattice QCD studies of the $\Lambda$ baryon have been attempted, most have used the quenched approximation, and none have identified an odd-parity state low enough in energy to be identified with the $\Lambda(1405)$. Our recent work has successfully isolated three low-lying states, and an extrapolation of the trend for the lowest-lying state to the physical pion mass reproduces the mass of the $\Lambda(1405)$ [2]. Subsequent work by the BGR Collaboration has confirmed these results [3]. To isolate these states, we used a correlation matrix analysis together with source and sink smearing, and these results are summarised in Figure 1. Using the same techniques, we can also investigate the electromagnetic structure of excited states, providing information about their internal structure.

We use the PACS-CS $(2+1)$-flavour full-QCD ensembles [ [4], available through the ILDG [5]. They are $32^{3} \times 64$ lattices with $\beta=1.90$. There are 5 pion masses available, ranging from $640 \mathrm{MeV}$ down to $156 \mathrm{MeV}$, and in the limit of physical pion mass, the lattice spacing is $0.0907(33) \mathrm{fm}$. The strange quark hopping parameter is fixed at $\kappa_{\mathrm{s}}=0.13640$, however this gives a kaon which is slightly too heavy, so we partially quench the strange quark sector by using $\kappa_{\mathrm{s}}=0.13665$ for the valence quarks. In these proceedings, we focus on the ensemble with the heaviest quark mass, with $\kappa_{\mathrm{u}, \mathrm{d}}=0.13700$, and have analysed 360 configurations. Statistical uncertainties are estimated via the jackknife method and best fits are selected by considering the full covariance-matrix-based reduced $\chi^{2}$.

In the following section we briefly recap the correlation matrix formalism, while in Section 3 we discuss the calculation and extraction of form factors. Finally, in Section 4 we present preliminary results of this analysis for the electric and magnetic Sachs form factors.

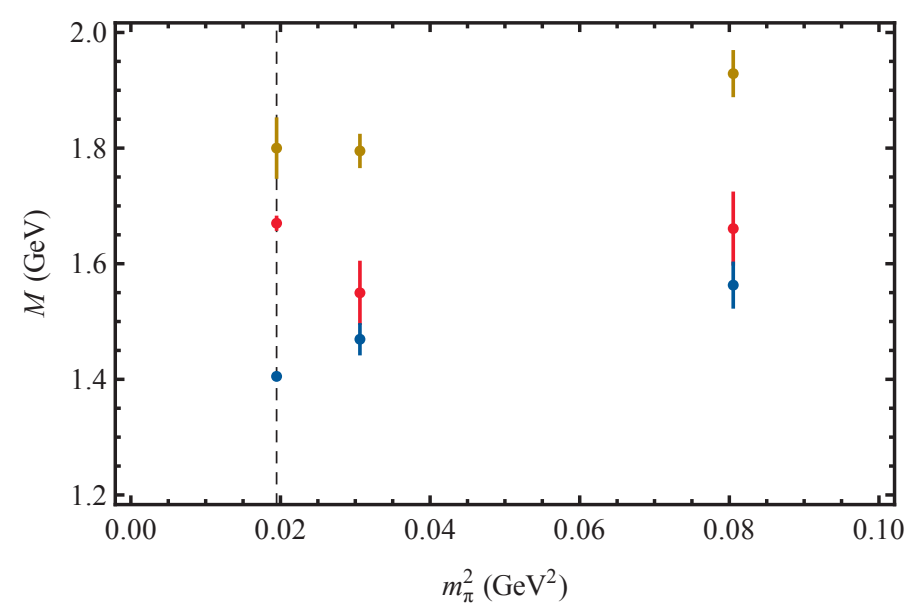

Figure 1: Masses of the three lowest-lying $J^{P}=1 / 2^{-}$states of the $\Lambda$ baryon, as found in [2]. An extrapolation to the physical pion mass of the trend for the lowest-lying state reproduces the correct physical mass of the $\Lambda(1405)$ indicated on the dashed line indicating the physical pion mass. 


\section{Correlation Matrix Technique}

Consider a set of $N$ operators $\chi_{i}(x)$ that couple to the baryon we are interested in, and calculate the $N \times N$ matrix of cross-correlation functions,

$$
\begin{aligned}
G_{i j}(\Gamma ; \mathbf{p} ; t) & =\sum_{\mathbf{x}} \mathrm{e}^{-\mathrm{i} \mathbf{p} \cdot \mathbf{x}} \operatorname{tr}\left(\Gamma\left\langle\Omega\left|\chi_{i}(x) \bar{\chi}_{j}(0)\right| \Omega\right\rangle\right) \\
& =\sum_{\alpha=1}^{N} Z_{i}^{\alpha}(\mathbf{p}) Z_{j}^{\alpha \dagger}(\mathbf{p}) \mathrm{e}^{-E_{\alpha}(\mathbf{p}) t} \operatorname{tr}\left(\Gamma \sum_{s} u^{\alpha}(p, s) \bar{u}^{\alpha}(p, s)\right)
\end{aligned}
$$

where $Z_{i}^{\alpha}(\mathbf{p})$ and $Z_{i}^{\alpha \dagger}(\mathbf{p})$ are the couplings of the operator $\chi_{i}$ to the state $\alpha$ at the sink and source, respectively. Now, construct a set of $N$ "perfect" operators $\phi^{\alpha}$ that completely isolate the $N$ lowestlying states, so that

$$
\left\langle\Omega\left|\phi^{\alpha}\right| \beta, p, s\right\rangle=\delta^{\alpha \beta} \mathscr{Z}^{\alpha}(\mathbf{p}) u(p, s) \quad \text { and } \quad\left\langle\beta, p, s\left|\bar{\phi}^{\alpha}\right| \Omega\right\rangle=\delta^{\alpha \beta} \mathscr{Z}^{\alpha^{\dagger}}(\mathbf{p}) \bar{u}(p, s),
$$

for some couplings $\mathscr{Z}^{\alpha}$ and $\mathscr{Z}^{\alpha \dagger}$. Using the linearity of the operator space, we can (approximately) write

$$
\phi^{\alpha}=\sum_{i} v_{i}^{\alpha}(\mathbf{p}) \chi_{i} \quad \text { and } \quad \bar{\phi}^{\alpha}=\sum_{i} u_{i}^{\alpha}(\mathbf{p}) \bar{\chi}_{i}
$$

The coefficient vectors $\mathbf{v}^{\alpha}$ and $\mathbf{u}^{\alpha}$ form the left and right generalised eigenvectors of the matrices $G\left(\Gamma ; \mathbf{p} ; t_{0}+\Delta t\right)$ and $G\left(\Gamma ; \mathbf{p} ; t_{0}\right)$ for some $t_{0}$ and $\Delta t$ :

$$
\begin{aligned}
G\left(\Gamma ; \mathbf{p} ; t_{0}+\Delta t\right) \mathbf{u}^{\alpha}(\mathbf{p}) & =\mathrm{e}^{-E_{\alpha} \Delta t} G\left(\Gamma ; \mathbf{p} ; t_{0}\right) \mathbf{u}^{\alpha}(\mathbf{p}) \\
\mathbf{v}^{\alpha \top}(\mathbf{p}) G\left(\Gamma ; \mathbf{p} ; t_{0}+\Delta t\right) & =\mathrm{e}^{-E_{\alpha} \Delta t} \mathbf{v}^{\alpha \top}(\mathbf{p}) G\left(\Gamma ; \mathbf{p} ; t_{0}\right) .
\end{aligned}
$$

Moreover, the coefficient vectors diagonalise the correlation matrix through

$$
\mathbf{v}^{\alpha \top}(\mathbf{p}) G(\Gamma ; \mathbf{p} ; t) \mathbf{u}^{\beta}(\mathbf{p}) \propto \delta^{\alpha \beta} \mathrm{e}^{-E_{\alpha} t},
$$

and hence we can define eigenstate-projected correlation functions that encode correlation functions for a single energy eigenstate as

$$
G^{\alpha}(\Gamma ; \mathbf{p} ; t):=\mathbf{v}^{\alpha \top}(\mathbf{p}) G(\Gamma ; \mathbf{p} ; t) \mathbf{u}^{\alpha}(\mathbf{p}) .
$$

More details about this method can be found in [2, 6, 7, 8, 9, 10].

To ensure the successful isolation of energy eigenstates, the original set of operators needs to be both large and span the space; too few operators and the states won't separate, while insufficiently independent operators will make the correlation matrix too ill-conditioned to solve the generalised eigensystem. To form our basis of operators we exploit both the various possible flavour symmetries for the $\Lambda$ and two different Dirac structures [6]. This gives a $3 \times 3$ matrix with the operators

$$
\chi_{i}^{8}=\frac{\varepsilon^{a b c}}{\sqrt{6}}\left(2\left(u_{a}^{\top} A_{i} d_{b}\right) B_{i} s_{c}+\left(u_{a}^{\top} A_{i} s_{b}\right) B_{i} d_{c}-\left(d_{a}^{\top} A_{i} s_{b}\right) B_{i} u_{c}\right),
$$

where $\left(A_{1}, B_{1}\right)=\left(C \gamma_{5}, \mathbb{I}\right)$ and $\left(A_{2}, B_{2}\right)=\left(C, \gamma_{5}\right)$, and

$$
\chi^{1}=-2 \varepsilon^{a b c}\left(-\left(u_{a}^{\top} C \gamma_{5} d_{b}\right) s_{c}+\left(u_{a}^{\top} C \gamma_{5} s_{b}\right) d_{c}-\left(d_{a}^{\top} C \gamma_{5} s_{b}\right) u_{c}\right) .
$$

We further increase the size of the operator basis employing gauge-invariant Gaussian smearing at the source and sink [11]. We use 16 and 100 sweeps of smearing with $\alpha=0.7$ to expand the matrix to a $6 \times 6$ one. 


\section{Form Factors of Negative Parity States}

To extract form factors we calculate both the two- and three-point correlation matrices

$$
\begin{aligned}
G_{i j}\left(\Gamma ; \mathbf{p}^{\prime} ; t_{2}\right) & =\sum_{\mathbf{x}_{2}} \mathrm{e}^{-\mathrm{i} \mathbf{p} \cdot \mathbf{x}_{2}} \operatorname{tr}\left(\Gamma\left\langle\Omega\left|\chi_{i}\left(x_{2}\right) \bar{\chi}_{j}(0)\right| \Omega\right\rangle\right) \\
G_{i j}^{\mu}\left(\Gamma ; \mathbf{p}^{\prime}, \mathbf{p} ; t_{2}, t_{1}\right) & =\sum_{\mathbf{x}_{1} \mathbf{x}_{2}} \mathrm{e}^{-\mathrm{i} \mathbf{p}^{\prime} \cdot \mathbf{x}_{2}} \mathrm{e}^{\mathrm{i}\left(\mathbf{p}^{\prime}-\mathbf{p}\right) \cdot \mathbf{x}_{1}} \operatorname{tr}\left(\Gamma\left\langle\Omega\left|\chi_{i}\left(x_{2}\right) j^{\mu}\left(x_{1}\right) \bar{\chi}_{j}(0)\right| \Omega\right\rangle\right),
\end{aligned}
$$

where $\chi_{i}$ is as before and $j^{\mu}$ is the electromagnetic current insertion. As before, we can project two-point correlation functions for individual energy eigenstates to give

$$
G_{\alpha}\left(\Gamma ; t_{2} ; \mathbf{p}^{\prime}\right):=\mathbf{v}^{\alpha \top}\left(\mathbf{p}^{\prime}\right) G\left(\Gamma ; t_{2} ; \mathbf{p}^{\prime}\right) \mathbf{u}^{\alpha}\left(\mathbf{p}^{\prime}\right),
$$

where $\mathbf{u}^{\alpha}$ and $\mathbf{v}^{\alpha}$ are the eigenvectors as in Equation (2.6).

Using the linearity of the operator space, Equation (2.3), if we act on the three-point correlation matrix (3.1) from the left and right by $\mathbf{v}^{\alpha}\left(\mathbf{p}^{\prime}\right)$ and $\mathbf{u}^{\alpha}(\mathbf{p})$, respectively, we obtain

$$
\begin{aligned}
\mathbf{v}^{\alpha \top}\left(\mathbf{p}^{\prime}\right) G_{i j}^{\mu}\left(\Gamma ; \mathbf{p}^{\prime}, \mathbf{p} ; t_{2}, t_{1}\right) \mathbf{u}^{\alpha}(\mathbf{p})= & \sum_{\mathbf{x}_{1}, \mathbf{x}_{2}} \mathrm{e}^{-\mathrm{i} \mathbf{p}^{\prime} \cdot \mathbf{x}_{2}} \mathrm{e}^{\mathrm{i}\left(\mathbf{p}^{\prime}-\mathbf{p}\right) \cdot \mathbf{x}_{1}} \times \\
& \times \operatorname{tr}\left(\Gamma \sum_{i, j}\left\langle\Omega\left|v_{i}^{\alpha}\left(\mathbf{p}^{\prime}\right) \chi_{i}\left(x_{2}\right) j^{\mu}\left(x_{1}\right) \bar{\chi}_{j}(0) u_{i}^{\alpha}(\mathbf{p})\right| \Omega\right\rangle\right) \\
= & \sum_{\mathbf{x}_{1}, \mathbf{x}_{2}} \mathrm{e}^{-\mathrm{i} \mathbf{p}^{\prime} \cdot \mathbf{x}_{2}} \mathrm{e}^{\mathrm{i}\left(\mathbf{p}^{\prime}-\mathbf{p}\right) \cdot \mathbf{x}_{1}} \operatorname{tr}\left(\Gamma\left\langle\Omega\left|\phi^{\alpha}\left(x_{2}\right) j^{\mu}\left(x_{1}\right) \bar{\phi}^{\alpha}(0)\right| \Omega\right\rangle\right) .
\end{aligned}
$$

Then, using the definition (2.2) of the "perfect" operators $\phi^{\alpha}$, we can see that this object encodes a three-point correlation function for the same energy-eigenstate as above:

$$
\begin{aligned}
\mathbf{v}^{\alpha \top}\left(\mathbf{p}^{\prime}\right) G_{i j}^{\mu}\left(\Gamma ; \mathbf{p}^{\prime}, \mathbf{p} ; t_{2}, t_{1}\right) \mathbf{u}^{\alpha}(\mathbf{p})= & \mathscr{Z}^{\alpha}\left(\mathbf{p}^{\prime}\right) \mathscr{Z}^{\alpha \dagger}(\mathbf{p}) \mathrm{e}^{-E_{\alpha}\left(\mathbf{p}^{\prime}\right)\left(t_{2}-t_{1}\right)} \mathrm{e}^{-E_{\alpha}(\mathbf{p}) t_{1}} \times \\
& \times \operatorname{tr}\left(\Gamma \sum_{s^{\prime}, s} u\left(p^{\prime}, s^{\prime}\right)\left\langle\alpha, p^{\prime}, s^{\prime}\left|j^{\mu}\left(x_{1}\right)\right| \alpha, p, s\right\rangle \bar{u}(p, s)\right),
\end{aligned}
$$

and hence we define this to be $G_{\alpha}^{\mu}$, an eigenstate-projected three-point correlation function. The matrix element in (3.4) can be written in the form

$$
\left\langle\alpha, p^{\prime}, s^{\prime}\left|j^{\mu}\left(x_{1}\right)\right| \alpha, p, s\right\rangle=\left(\frac{m_{\alpha}^{2}}{E_{\alpha}\left(\mathbf{p}^{\prime}\right) E_{\alpha}(\mathbf{p})}\right)^{1 / 2} \bar{u}\left(p^{\prime}, s^{\prime}\right)\left(F_{1}\left(q^{2}\right) \gamma^{\mu}+\mathrm{i} F_{2}\left(q^{2}\right) \frac{\sigma^{\mu v} q_{v}}{2 m_{\alpha}}\right) u(p, s),
$$

where $q:=p^{\prime}-p$ is the momentum transferred to the hadron in the interaction and $F_{1}$ and $F_{2}$ are the Dirac and Pauli form factors, related to the Sachs form factors through

$$
\mathscr{G}_{\mathrm{E}}^{\alpha}=F_{1}\left(q^{2}\right)-\frac{q^{2}}{\left(2 m_{\alpha}\right)^{2}} F_{2}\left(q^{2}\right) \quad \text { and } \quad \mathscr{G}_{\mathrm{M}}^{\alpha}=F_{1}\left(q^{2}\right)+F_{2}\left(q^{2}\right)
$$

To eliminate the time dependence in the three-point correlation function, we construct the ratio

$$
R_{\alpha}^{\mu}\left(\Gamma_{2}, \Gamma_{1} ; t_{2}, t_{1} ; \mathbf{p}^{\prime}, \mathbf{p}\right):=\left(\frac{G_{\alpha}^{\mu}\left(\Gamma_{1} ; \mathbf{p}^{\prime}, \mathbf{p} ; t_{2}, t_{1}\right) G_{\alpha}^{\mu}\left(\Gamma_{1} ; \mathbf{p}, \mathbf{p}^{\prime} ; t_{2}, t_{1}\right)}{G_{\alpha}\left(\Gamma_{2} ; \mathbf{p}^{\prime} ; t_{2}\right) G_{\alpha}\left(\Gamma_{2} ; \mathbf{p} ; t_{2}\right)}\right)^{1 / 2} .
$$


We can then further simplify the equation by constructing the reduced ratio

$$
\bar{R}_{\alpha}^{\mu}\left(\Gamma_{2}, \Gamma_{1} ; t_{2}, t_{1} ; \mathbf{p}^{\prime}, \mathbf{p}\right):=\sqrt{\frac{2 E_{\alpha}(\mathbf{p})}{E_{\alpha}(\mathbf{p})+m_{\alpha}}} \sqrt{\frac{2 E_{\alpha}\left(\mathbf{p}^{\prime}\right)}{E_{\alpha}\left(\mathbf{p}^{\prime}\right)+m_{\alpha}}} R_{\alpha}^{\mu}\left(\Gamma_{2}, \Gamma_{1} ; t_{2}, t_{1} ; \mathbf{p}^{\prime}, \mathbf{p}\right) .
$$

Now, a suitable choice of $\Gamma_{1}$ and $\Gamma_{2}$ allows us to directly extract the Sachs form factors for positiveparity states $\alpha[12$ :

$$
\begin{aligned}
\mathscr{G}_{\mathrm{E}}^{\alpha}\left(q^{2}\right) & =\bar{R}_{\alpha}^{4}\left(\Gamma_{4}^{+}, \Gamma_{4}^{+} ; \mathbf{q}, \mathbf{0}\right) \text { and } \\
\left|\varepsilon_{i j k} q^{i}\right| \mathscr{G}_{\mathrm{M}}^{\alpha}\left(q^{2}\right) & =\left(E_{\alpha}(\mathbf{q})+m_{\alpha}\right) \bar{R}_{\alpha}^{k}\left(\Gamma_{4}^{+}, \Gamma_{j}^{+} ; \mathbf{q}, \mathbf{0}\right),
\end{aligned}
$$

where

$$
\Gamma_{j}^{+}=\frac{1}{2}\left[\begin{array}{cc}
\sigma_{j} & 0 \\
0 & 0
\end{array}\right] \quad \text { and } \quad \Gamma_{4}^{+}=\frac{1}{2}\left[\begin{array}{cc}
\mathbb{I} & 0 \\
0 & 0
\end{array}\right]
$$

For negative-parity states, we repeat the analysis after making the substitutions $\chi_{i} \mapsto \gamma_{5} \chi_{i}$ and $\bar{\chi}_{i} \mapsto$ $-\bar{\chi}_{i} \gamma_{5}$ to obtain the equivalent negative-parity operators. Using the cyclic nature of the trace, we see that the equivalent projection operators for negative-parity states are then

$$
\Gamma_{j}^{-}=-\gamma_{5} \Gamma_{j}^{+} \gamma_{5}=-\frac{1}{2}\left[\begin{array}{cc}
0 & 0 \\
0 & \sigma_{j}
\end{array}\right] \text { and } \Gamma_{4}^{-}=-\gamma_{5} \Gamma_{4}^{+} \gamma_{5}=-\frac{1}{2}\left[\begin{array}{ll}
0 & 0 \\
0 & \mathbb{I}
\end{array}\right] .
$$

\section{First Results}

As demonstrated in Figures 2 and 3 , this technique allows us to successfully extract the form factors for negative-parity excited states. Indeed, we can often begin fitting only one time slice after the insertion of the current at $t_{1}=21$. Moreover, due to the correlated nature of the statistical errors in the two- and three-point correlation functions, by taking the ratio in Equation (3.8) we obtain signals that last for many time slices, allowing for long and precise fits. The $q^{2}$ is $0.14224(4) \mathrm{GeV}^{2}$ for the $\Lambda(1405)$.

The electric form factor for individual quark flavours for the $\Lambda(1405)$, illustrated in Figure 2 , are almost identical to those of the positive-parity ground-state $\Lambda$. This indicates that the quark distributions are essentially the same between the $\Lambda$ and $\Lambda(1405)$, and hence there is no evidence of a more exotic structure for the $\Lambda(1405)$ (in particular, a bound meson-baryon structure would generally manifest as a larger quark distribution radii, and hence smaller form factors). This close proximity of the quarks to the origin in an orbitally excited state is remarkable. In a potential model, this suggests that the flavour-singlet potential is particularly attractive; the quarks would feel the depth of the potential and the system would have an unusually low excitation energy.

For the second and third odd-parity excited states, the electric form factors are significantly smaller, indicating larger states. The magnetic form factor for these two states has a large contribution from the strange quark and hence the charge-weighted sum is non-zero and negative. This structure is similar to that of the octet ground state $\Lambda$. For the $\Lambda(1405)$ however, this structure is not evident; all three quark flavours have approximately the same unit-charge-scaled $\mathscr{G}_{\mathrm{M}}$, and so the total charge-weighted magnetic form factor vanishes within errors. 

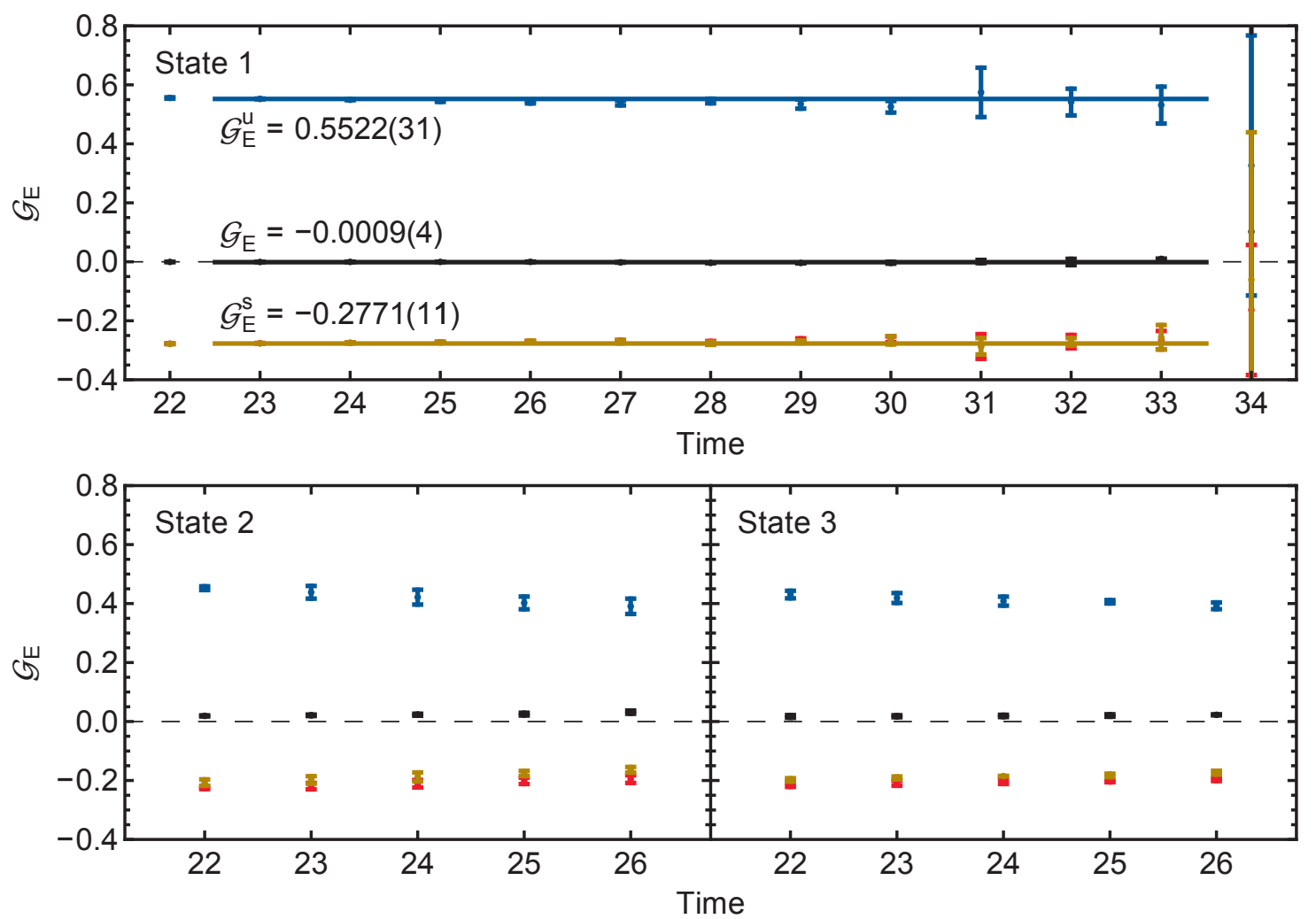

Figure 2: Sachs electric form factor $\mathscr{G}_{\mathrm{E}}$ of charge-scaled quark sectors for the three lowest-lying states. Blue is up quark, red is down quark, gold is strange quark, and black is charge-weighted sum. Due to isospin symmetry, the fit (not shown) to the down quark is $-1 / 2$ that of the up quark.

\section{Acknowledgments}

This research was undertaken with the assistance of resources at the NCI National Facility in Canberra, Australia, and the iVEC facilities at Murdoch University (iVEC@Murdoch) and the University of Western Australia (iVEC@UWA). These resources were provided through the National Computational Merit Allocation Scheme, supported by the Australian Government. This research is supported by the Australian Research Council.

\section{References}

[1] J. Beringer et al. (Particle Data Group), Review of Particle Physics, Phys. Rev. D 86 (2012) 010001.

[2] B. J. Menadue, W. Kamleh, D. B. Leinweber, and M. S. Mahbub, Isolating the A(1405) in Lattice QCD, Phys. Rev. Lett. 108 (2012) 112001 [1109.6716].

[3] G. P. Engel, C. B. Lang, and A. Schäfer, Low-lying Lambda Baryons from the Lattice (2012) [1212.2032].

[4] S. Aoki et al. (PACS-CS Collaboration), 2+1 Flavor Lattice QCD toward the Physical Point, Phys. Rev. D 79 (2009) 034503 [0807.1661].

[5] M. G. Beckett, P. Coddington, B. Joó, C. M. Maynard, D. Pleiter, O. Tatebe, and T. Yoshie, Building the International Lattice Data Grid, Comput. Phys. Commun. 182 (2011) 1208 [0910 .1692]. 

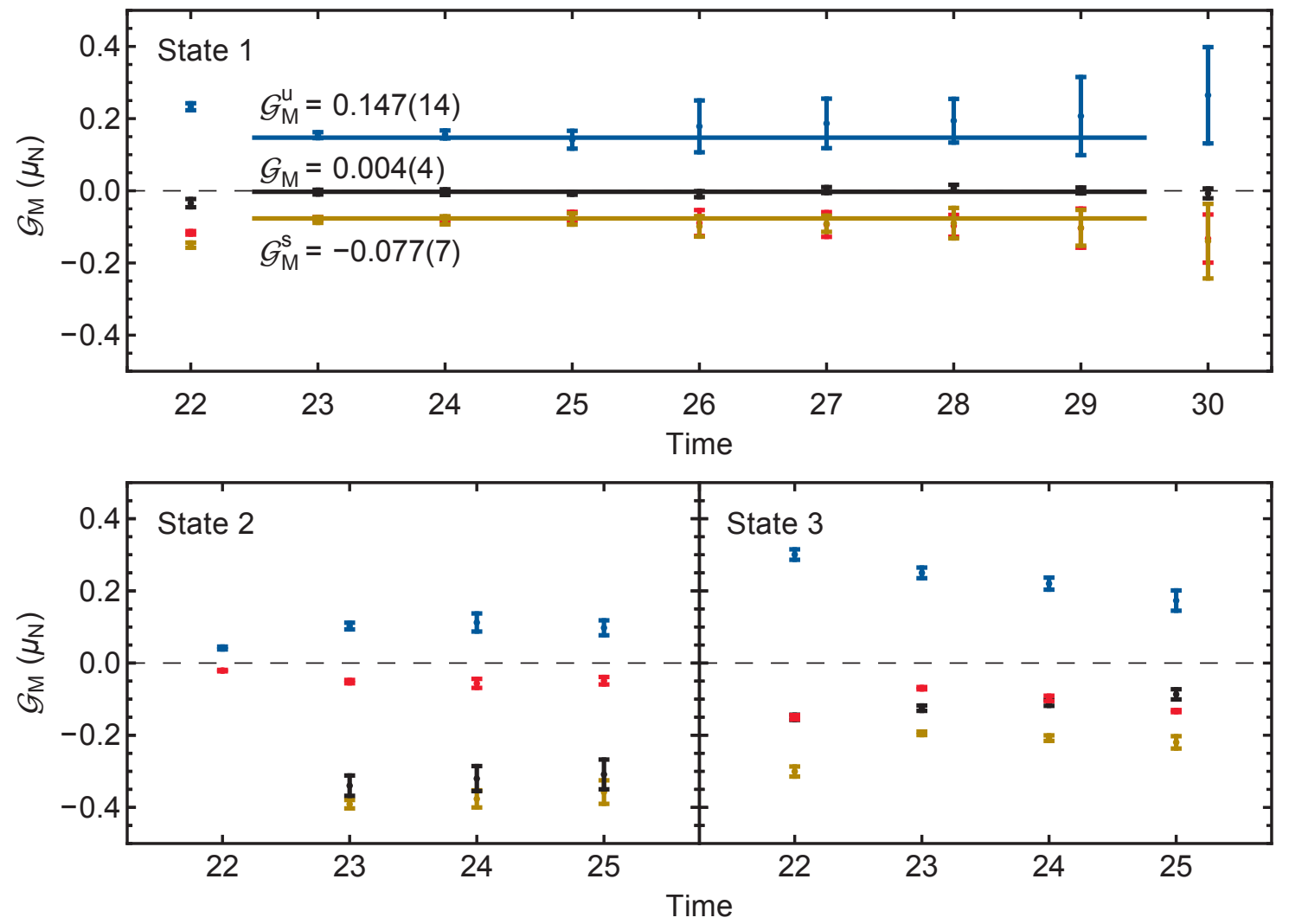

Figure 3: Sachs magnetic form factor $\mathscr{G}_{\mathrm{M}}$ in units of nuclear magnetons of charge-scaled quark sectors for the three lowest-lying states. Blue is up quark, red is down quark, gold is strange quark, and black is charge-weighted sum. Due to isospin symmetry, the fit (not shown) to the down quark is $-1 / 2$ that of the up quark.

[6] D. B. Leinweber, W. Melnitchouk, D. G. Richards, A. .G. Williams, and J. M. Zanotti, Baryon Spectroscopy in Lattice QCD, Lect. Notes Phys. 663 (2005) 71 [nucl - th/0406032].

[7] M. S. Mahbub, A. Ó. Cais, W. Kamleh, B. G. Lasscock, D. B. Leinweber, and A. G. Williams, Isolating Excited States of the Nucleon in Lattice QCD, Phys. Rev. D 80 (2009) 054507 [0905.3616].

[8] M. S. Mahbub, A. Ó. Cais, W. Kamleh, D. B. Leinweber, and A. G. Williams, Positive-Parity Excited States of the Nucleon in Quenched Lattice QCD, Phys. Rev. D 82 (2010) 094504 [1004 . 5455].

[9] B. J. Menadue, W. Kamleh, D. B. Leinweber, and M. S. Mahbub, Extracting Low-Lying Lambda Resonances Using Correlation Matrix Techniques, AIP Conf. Proc. 1354 (2011) 213 [1102 . 3492 ].

[10] M. S. Mahbub, W. Kamleh, D. B. Leinweber, P. J. Moran, and A. G. Williams, Roper Resonance in 2+1 Flavor QCD, Phys. Lett. B 707 (2012) 389 [1011.5724].

[11] Güsken, S, A Study of Smearing Techniques for Hadron Correlation Functions, Nucl. Phys. B (Proc. Suppl.) 17 (1990) 361.

[12] D. B. Leinweber, R. M. Woloshyn, and T. Draper, Electromagnetic Structure of Octet Baryons, Phys. Rev. D 43 (1991) 1659. 